\title{
Onconase downregulates microRNA expression through targeting microRNA precursors
}

Cell Research (2012) 22:1199-1202. doi:10.1038/cr.2012.67; published online 24 April 2012

\section{Dear Editor,}

Onconase, the smallest member of RNase A super family that was isolated from oocytes or early embryos of the Northern Leopard Frog (Rana pipiens), has been granted as an orphan drug for the treatment of malignant mesothelioma by US FDA $[1,2]$. It was also tested in clinical trials for patients with non-small-cell lung cancer, breast cancer, and renal cell cancer [2]. Onconase is extremely stable with a $\mathrm{Tm}$ of $\sim 87^{\circ} \mathrm{C}$, resists degradation by various proteases, and evades ribonulease inhibitors (RI) present in mammalian cell cytosol [3-5], which confers advantages to its application in cancer treatment. More importantly, onconase specifically induces apoptosis of cancer cells but has low cytotoxicity to their normal counterparts [2]. One rational hypothesis for this selectivity is that onconase, a highly cationic protein with calculated $\mathrm{pI}>9.5$, is selectively internalized by tumor cells given that tumor cells generally are more negatively charged than cognate normal cells [2]. Nevertheless, as an anti-cancer drug, the mechanisms of its antitumor activity are not well understood. The current model of onconase-mediated cytotoxicity favors that onconase degrades tRNAs in tumor cells after its cytosolic internalization, which leads to ubiquitous inhibition of protein synthesis and apoptosis [2]. However, increasing evidence indicates that degradation of tRNAs and inhibition of protein synthesis are not the sole cause of onconase-induced apoptosis [6-8]. Consistent with the welldocumented causal roles of microRNAs (miRNAs) in cancers, a recent study indicates that onconase regulates the expression of miRNAs in malignant pleural mesothelioma cell lines (H2959, H2373, and H2591), and reveals that onconase controls cell proliferation, invasion, migration, and apoptosis through modulating miRNAs [9]. However, how onconase affects miRNA expression remains unclear. Here our biochemical studies showed that miRNAs are the direct downstream RNA species of onconase. Intriguingly, we found that onconase downregulates miRNAs by cleavage of miRNA precursors, thus reducing the amount of mature miRNAs produced from
Dicer processing.

Using recombinant onconase that was prepared as described previously [10], we found that miR-155 and miR-21, two well-known oncogenic miRNAs with high endogenous levels in mesothelioma cell line Msto-211h, were dose- and time-dependently downregulated by onconase in these cells (Supplementary information, Figure S1A, S1B, S1D and S1E), accompanied by a significant upregulation of their respective targets examined, including miR-155 targets SOCS1 [11], C/EBP $\beta[12,13]$, and ETS-1 [14], and miR-21 targets PTEN [15] and PDCD4 [16] (Supplementary information, Figure S1C and S1F). Furthermore, our miRNA microarray assays showed that the majority of miRNAs in Msto-211h cells were significantly downregulated by onconase (Supplementary information, Figure S2A), while our quantitative realtime PCR (qRT-PCR) assays validated the onconasemediated downregulation of let-7 family miRNAs, miR21, miR-29a, miR-92a, miR-92b, miR-155, miR-221, and miR-222 (Supplementary information, Figure S2B). These results indicate that miRNA expression is susceptible to onconase, consistent with the recent report [9]. However, different from the findings of upregulation of 5 miRNAs in addition to downregulation of the majority in mesothelioma cells by onconase in that study [9], we found that onconase appears to globally downregulate miRNAs in Msto-211h cells. This dissimilarity is likely due to a different cell line examined in this study. Nevertheless, our microarray assays also showed that four miRNAs (miR-181a, miR-30a, miR-1280, and miR-720) were upregulated by onconase treatment (Supplementary information, Figure S2A); however, further analyses revealed that all these four miRNAs showed low intensities in microarray assays (Supplementary information, Figure S2C). Moreover, our qRT-PCR assays showed that miR-181a expression was actually reduced $>2$-fold by onconase treatment (Supplementary information, Figure S2B), while the other three miRNAs were undetectable in qRT-PCR assays (data not shown), implicating low endogenous levels of these miRNAs in Msto-211h cells and false positive in microarray assays. It is noteworthy 

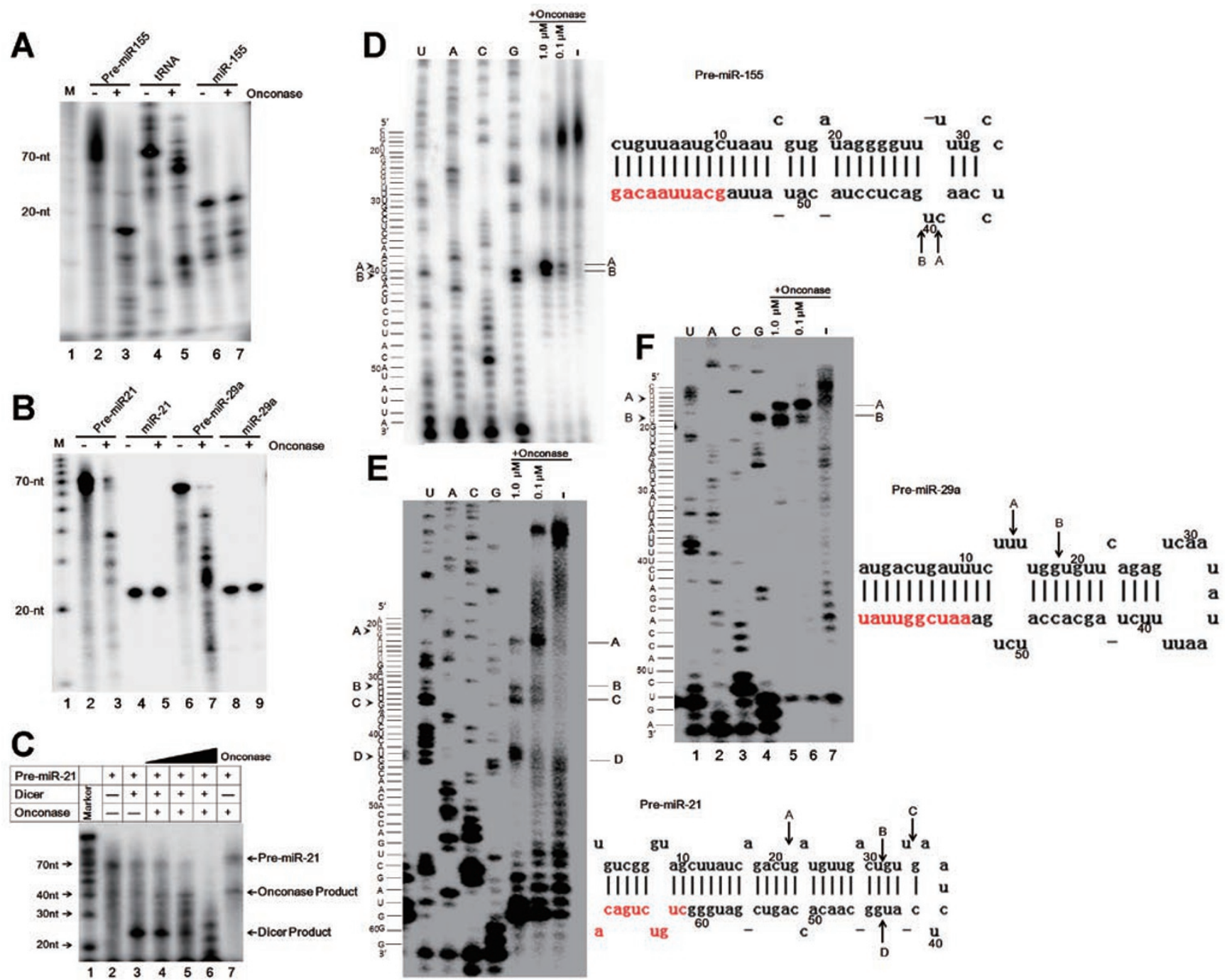

Figure 1 Onconase downregulates miRNA expression by targeting miRNA precursors. (A, B) In vitro onconase reactions to examine the effect of onconase on mature and precursor forms of miRNAs. (A) The effect of onconase on miR-155. (B) The effect of onconase on miR-21 and miR-29a. M, RNA markers. (C) Processing of pre-miR-21 into mature miR-21 by Dicer in vitro was dose-dependently reduced by onconase. Lane 1, RNA markers; lane 2, miR-21 precursor alone; lane 3, Dicer and miR-21 precursor; lanes 4-6, Dicer, miR-21 precursor, and increasing amounts of onconase $(0.1,0.5$, and $2.5 \mu \mathrm{M})$; lane 7 , oncoanse $(0.1 \mu \mathrm{M})$ and miR-21 precursor. (D-F) Primer extension analyses of the cleavage sites of onconase in miR-155 precursor (D), miR-21 precursor (E), and miR-29a precursor (F). Lanes 1-4, DNA sequencing of miRNA precursors; lanes $5-7$, determination of the positions of cleavage with $1.0 \mu \mathrm{M}$ and $0.1 \mu \mathrm{M}$ onconase, and without onconase, respectively. Arrows indicate positions of cleavages. The sequences that were recognized by primers in both of DNA sequencing and primer extension assays are shown in red.

that 4 out of 5 upregulated miRNAs have extremely low values of intensities in their miRNA microarray data [9], while all of these five upregulated miRNAs do not meet the cutoff of mean intensities above 500 in our microarray assays.

We next asked how onconase downregulates miRNAs. To this end, we performed in vitro onconase reactions to examine the effect of onconase on chemically syn- thesized 23-nt mature miR-155 and 65-nt pre-miR-155 strands (Supplementary information, Data S1). A human tRNA ${ }^{\text {Arg }}$, which was transcribed by T7 RNA polymerase in vitro, was used as a positive control for onconase activity. We found that this tRNA strand was strongly degraded by onconase (Figure 1A, lane 5), indicating that onconase prepared in the lab is highly active. Interestingly, we found that miR-155 precursor strand was 
significantly cleaved by onconase (Figure 1A, lane 3), while mature miR-155 strand was marginally affected (Figure 1A, lane 7), suggesting that onconase preferentially degrades the precursor forms of miRNAs. To further corroborate this, we examined the effect of onconase on other miRNAs. Consistently, we found that onconase strongly degraded the precursor strands of miR-21 and miR-29a, but mildly affected their mature forms (Figure 1B). These results together indicate that oncoanse preferentially degrades miRNA precursors instead of the mature forms of miRNAs.

Given that onconase preferably cleaves miRNA precursors (Figure 1A and 1B) and that miRNA precursors are processed into miRNAs by Dicer in cells, we speculated that onconase might reduce miRNA expression through disturbing Dicer-mediated mature miRNA production in cancer cells. Indeed, using in vitro Dicer processing analyses (Supplementary information, Data $\mathrm{S} 1$ ), we found that processing of pre-miR-21 into mature miR-21 by Dicer was dose-dependently reduced by onconase (Figure 1C). These results suggest that onconase degrades miRNA precursors and subsequently results in less production of mature miRNAs produced by Dicer.

Finally, we mapped the cleavage sites of onconase in miRNA precursors. Using primer extension analyses (Supplementary information, Data S1), we found that pre-miR-155 was mainly cleaved at $\mathrm{C}^{39}-\mathrm{U}^{40}$ and $\mathrm{U}^{40}-\mathrm{G}^{41}$ (Figure 1D), pre-miR-21 at $U^{21}-G^{22}, U^{31}-G^{32}, U^{34}-G^{35}$, and $U^{43}-G^{44}$ (Figure 1E), and pre-miR-29a at $U^{14}-U^{15}$ and $\mathrm{U}^{18}-\mathrm{G}^{19}$ (Figure $1 \mathrm{~F}$ ). These results indicate that onconase appears to predominantly cleave miRNA precursors at UG and UU residues, which are similar to the cleavage specificity of onconase observed in tRNAs [17].

In summary, our results indicate that onconase ubiquitously downregulates miRNA expression in mesothelioma cells. Interestingly, our biochemical assays reveal that onconase preferentially degrades miRNA precursors and mildly affects mature forms of miRNAs. Given that miRNA precursors resemble tRNAs, the well-documented downstream RNA species of onconase, with $\sim 70$-nt long and hairpin structure, we speculate that an appropriate secondary structure might be required for onconase substrates. Indeed, similar to the predominant cleavages of onconase in tRNAs located in the variable loop or stem of D-arm [17], we found that the cleavage sites of onconase in miRNA precursors are mapped at both loop and stem regions. Given that oncogenic miRNAs are upregulated in cancer cells while tumor suppressive miRNAs are often downregulated [18], it is likely that onconase exerts its antitumor activity through targeting miRNAs, i.e., mainly oncogenic miRNAs. Taken together, our study reveals miRNA precursors as a novel class of RNA targets for onconase in addition to tRNAs, bringing new insights into the mechanisms of onconasemediated cytotoxicity in cancer cells.

\section{Acknowledgments}

We thank Prof En-Duo Wang (Institute of Biochemistry and Cell Biology, SIBS, CAS) for providing human tRNA ${ }^{\text {Arg }}$. This work was supported by grants from the Ministry of Science and Technology of China (2011CB811303, 2012CB910802, 2011CB966304) and the National Natural Science Foundation of China (30970621, 31170754).

Meng Qiao ${ }^{1,2,3, *}$, Li-Dong $\mathrm{Zu}^{1,2,3, *}$, Xiao-Hong $\mathrm{He}^{1,2,3}$, Ru-Ling Shen ${ }^{4}$, Qing-Cheng Wang ${ }^{4}$, Mo-Fang Liu ${ }^{1,2,3}$

${ }^{I}$ State Key Laboratory of Molecular Biology, Graduate School of Chinese Academy of Sciences, Shanghai 200031, China; ${ }^{2}$ Shanghai Key Laboratory of Molecular Andrology, Shanghai 200031, China; ${ }^{3}$ Center for RNA Research, Institute of Biochemistry and Cell Biology, Shanghai Institutes for Biological Sciences, Chinese Academy of Sciences, Shanghai 200031, China; ${ }^{4}$ Shanghai Research Center for Model Organisms, Shanghai 201210, China

*These two authors contributed equally to this work.

Correspondence: Mo-Fang Liu

Tel: +86-21-54921146; Fax: +86-21-54921101

E-mail: mfliu@sibs.ac.cn

\section{References}

1 Pavlakis N, Vogelzang NJ. Ranpirnase--an antitumour ribonuclease: its potential role in malignant mesothelioma. Expert Opin Biol Ther 2006; 6:391-399.

2 Lee JE, Raines RT. Ribonucleases as novel chemotherapeutics: the ranpirnase example. BioDrugs 2008; 22:53-58.

3 Notomista E, Catanzano F, Graziano G, et al. Onconase: an unusually stable protein. Biochemistry 2000; 39:8711-8718.

4 Haigis MC, Kurten EL, Raines RT. Ribonuclease inhibitor as an intracellular sentry. Nucleic Acids Res 2003; 31:1024-1032.

5 Dickson KA, Haigis MC, Raines RT. Ribonuclease inhibitor: structure and function. Prog Nucleic Acid Res Mol Biol 2005; 80:349-374.

6 Juan G, Ardelt B, Li X, et al. G1 arrest of U937 cells by onconase is associated with suppression of cyclin D3 expression, induction of p16INK4A, p21WAF1/CIP1 and p27KIP and decreased pRb phosphorylation. Leukemia 1998; 12:1241-1248.

7 Iordanov MS, Ryabinina OP, Wong J, et al. Molecular determinants of apoptosis induced by the cytotoxic ribonuclease onconase: evidence for cytotoxic mechanisms different from inhibition of protein synthesis. Cancer Res 2000; 60:19831994.

8 Altomare DA, Rybak SM, Pei J, et al. Onconase responsive genes in human mesothelioma cells: implications for an RNA damaging therapeutic agent. BMC Cancer 2010; 10:34.

9 Goparaju CM, Blasberg JD, Volinia S, et al. Onconase mediat- 
ed NFK $\beta$ downregulation in malignant pleural mesothelioma. Oncogene 2011; 30:2767-2777.

10 Shen RL, Sun RL, Wang QC, Ou L, Fei J. Growth inhibition effect of onconase on B16 melanoma cells in vivo and in vitro. Chinese J Cell Biol 2007; 29: 901-904.

11 Jiang S, Zhang HW, Lu MH, et al. MicroRNA-155 functions as an OncomiR in breast cancer by targeting the suppressor of cytokine signaling 1 gene. Cancer Res 2010; 70:3119-3127.

12 Costinean S, Sandhu SK, Pedersen IM, et al. Src homology 2 domain-containing inositol-5-phosphatase and CCAAT enhancer-binding protein beta are targeted by miR-155 in B cells of Emicro-MiR-155 transgenic mice. Blood 2009; 114:13741382.

13 He M, Xu Z, Ding T, Kuang DM, Zheng L. MicroRNA-155 regulates inflammatory cytokine production in tumor-associated macrophages via targeting C/EBPbeta. Cell Mol Immunol 2009; 6:343-352.
14 Romania P, Lulli V, Pelosi E, Biffoni M, Peschle C, Marziali G. MicroRNA 155 modulates megakaryopoiesis at progenitor and precursor level by targeting Ets-1 and Meis 1 transcription factors. Br J Haematol 2008; 143:570-580.

15 Meng F, Henson R, Wehbe-Janek H, Ghoshal K, Jacob ST, Patel T. MicroRNA-21 regulates expression of the PTEN tumor suppressor gene in human hepatocellular cancer. Gastroenterology 2007; 133:647-658

$16 \mathrm{Lu} \mathrm{Z}$, Liu M, Stribinskis V, et al. MicroRNA-21 promotes cell transformation by targeting the programmed cell death 4 gene. Oncogene 2008; 27:4373-4379.

17 Suhasini AN, Sirdeshmukh R. Transfer RNA cleavages by onconase reveal unusual cleavage sites. J Biol Chem 2006; 281:12201-12209.

18 Kasinski AL, Slack FJ. Epigenetics and genetics. MicroRNAs en route to the clinic: progress in validating and targeting microRNAs for cancer therapy. Nat Rev Cancer 2011; 11:849-864.

(Supplementary information is linked to the online version of the paper on the Cell Research website.) 